\title{
Diesel Microemulsion Fuel Resistant to Low-temperature Conditions: Study of Thermal Stability and Breakup
}

\author{
Alexander Ashihmin ${ }^{1}$, Maxim Piskunov ${ }^{1}$, Vyacheslav Yanovsky ${ }^{2}$ \\ ${ }^{1}$ National Research Tomsk Polytechnic University \\ 30, Lenin Ave., Tomsk, Russia \\ alashikhmin24@gmail.com; piskunovmv@tpu.ru \\ ${ }^{2}$ National Research Tomsk State University \\ 36, Lenin Ave., Tomsk, Russia \\ yavatpu@yandex.ru
}

\begin{abstract}
The study was performed by using the alternative fuel prepared by the authors - water-in-diesel or ammonium acetate brinein-diesel microemulsions (WDME or BDME) stabilized by a non-ionic surfactant and various alcohols with a carbon chain length from $\mathrm{C}_{5}$ to $\mathrm{C}_{9}$ as co-surfactants. The study provides results on thermal stability of the fuel mentioned and a breakup of its drops during the impact onto a horizontal heated wall. The processes under study are crucial for developing advanced fuel technologies intended to reach conditions of the enhanced fuel vaporization, ignition, and combustion inside combustion chambers of various IC-engines and power generation systems. Learning of the thermal stability involves several ways for controlling temperature ranges of the prepared fuel existence. Among of them are a utilization of the ammonium acetate brines as a dispersed phase, as well as alcohols (hexanol-1, 2ethylhexanol, nonanol-1) as a co-surfactant when stabilizing microemulsions, a variation of the co-surfactant fraction in a surfactant/cosurfactant mixture (emulsifier), as well as the content of the dispersed phase relative to the continuous one. By using these ways we succeeded in fabricating the microemulsion fuel samples stable to phase separation in a temperature range from about $-20^{\circ} \mathrm{C}$ to $+60^{\circ} \mathrm{C}$. For physical modeling of drop impingement of the fabricated fuels onto heated surfaces of a combustion chamber, we investigated hydrodynamic regimes of single drop interaction with a horizontal wall. In addition, regime maps were plotted, and the determining relationships between inertia, viscosity and surface tension forces were established. The study presents critical Weber and Reynolds numbers characterizing the thermal breakup/rebound transition. An application of the alternative fuels is of the great interest because it is possible to enhance considerably an explosive breakup of drops in combustion chambers due to low-boiling component vaporization. Such an effect enables a significant increase in fuel burnout and the reduction of environmentally harmful emissions.
\end{abstract}

Keywords: alternative fuel, microemulsion, diesel, stability, drop breakup, drop rebound

\section{Introduction}

Diesel is used globally in various engines and power generation systems. However, while diesel burning, as known, many environmentally hazardous emissions release, namely nitrogen oxides $\left(\mathrm{NO}_{\mathrm{x}}\right)$, carbon monoxide $(\mathrm{CO})$, hydrocarbons (HC), particulate matters (PM) etc. One of the variants to solve this problem is to supplement water in the neat diesel; such a way promotes to a decrement of emissions [1]. A fabrication of water-in-diesel emulsions and their utilization as a fuel alternative to diesel is another way for decreasing emissions and solving a number of other tasks associated with an operation of various diesel engines [2,3]. Among of such tasks are the attainment of the minimum ignition delay, as well as enhanced fuel vaporization and burnout. Nevertheless, the water-in-diesel emulsions are unstable tends to phase separation after sometimes. A preparation of water-in-diesel microemulsions (WDME) solves fuel instability problem. Such liquids can remain in a single-phase state for a long time. Moreover, there are studies demonstrating the applicability of these microemulsions as an alternative more ecologically friendly fuel for use in various diesel engines [4,5]. However, the WDME stability control in conditions of a fluctuating environment temperature is almost unexplored. Only recently, some studies become to consider the thermal stability of microemulsions at temperatures different from standard one [4,6,7]. Alongside this, we did not find researches of the thermal stability control of the WDME fuels under low and subzero temperatures. Thus, a fabrication of the WDME fuels with cloud points similar to the neat diesel is a promising task. Along with that, learning of impact hydrodynamics of single drops of the fabricated stable WDME fuels is one of the initial fundamental steps for physical modeling of the behavior of such drops in real conditions. 
In this context, the purpose of the study is (1) to establish ways for controlling the thermal stability of the WDME fuels in temperature conditions different from standard ones; (2) to learn an impingement of the single drop of the WDME fuel stable in a wide range of environment temperatures onto a horizontal heated wall.

\section{Materials and Methods}

\subsection{WDME fuel: blend composition}

To prepare the microemulsion fuel, the following components were used: continuous phase - winter diesel fuel (EURO, class 3, type I-III (winter) in accordance with the Russian State Standard GOST R 52368-2005, density at $25^{\circ} \mathrm{C}$ $\rho=830 \mathrm{~kg} / \mathrm{m}^{3}$, kinematic viscosity at $25^{\circ} \mathrm{C} v=2.78 \cdot 10^{-6} \mathrm{~m}^{2} / \mathrm{s}$, surface tension $\left.\sigma=0.0291 \mathrm{~N} / \mathrm{m}\right)$, dispersed phase distilled water with a conductivity of no more than $5 \mu \mathrm{S} / \mathrm{cm}$ and ammonium acetate (reagent grade, GOST 3117-78); surfactant Neonol AF 9-6, PEG-6 nonylpheny lether (TU 2483-077-05766801-98, PJSC Nizhnekamskneftekhim, Russia), co-surfactant - 2-ethylhexanol (industrial, Russian State Standard GOST 26624-2016), hexanol-1 (reagent grade, Merck), isoamyl alcohol (reagent grade, Russian State Standard GOST 5830-79), and nonanol-1 (reagent grade, Merck). A volume concentration of the surfactant/co-surfactant $(S / c S)$ mixture ranged from 9.9 vol\% to $41.2 \mathrm{vol} \%$. The term "WDME fuel" implies a homogeneous optically transparent system often named as Winsor Type IV. To fabricate the WDME fuel, a magnetic mixer Daihan Scientific MSH-20D was applied. A thermal stability testing was performed by using a liquid low-temperature thermostat. For each of samples, we measured the upper and lower cloud points (CPs) localizing a temperature band of a single-phase state of the WDME fuel. CPs were determined visually in heating and cooling modes. For the upper $\mathrm{CP}$, the heating mode corresponded to a transition from a single-phase state (transparent liquid) to a two-phase one (emulsion), but the cooling mode - two-phase / single-phase transition. For the lower $\mathrm{CP}$, observations were opposite. An error in measuring equaled to $\pm 0.1^{\circ} \mathrm{C}$.

The study included a variation of the ratios of microemulsion fuel components, namely diesel / distilled water $(D / W)$ and surfactant / co-surfactant $(S / c S)$. To find out an effect of the nature of alcohols used as co-surfactants on temperature ranges of the WDME fuel stability, we applied hexanol-1, 2-ethylhexanol, nonanol-1. In addition, we assumed that the $S / c S$ ratio could influence the thermal stability. This ratio varied as follows: $10 / 0,9 / 1$, and $7 / 3$. Moreover, we believed that the use of brines as a dispersed phase would promote to changing in temperature ranges of stability. The microemulsion fuels fabricated using brines were titled as brine-in-diesel microemulsion fuel (BDME fuel). The $0.1 \mathrm{M}$ and $1 \mathrm{M}$ ammonium acetate brines were used as a dispersed phase.

\subsection{WDME fuel drop impact onto a heated surface}

The study of the microemulsion drop impact onto a horizontal heated wall was performed by the set-up depicted in Fig. 1. This set-up is typical and applied in a number of works with some upgrading [8,9]. The main elements of the setup are the $1.8 \mathrm{~kW}$ heating plate, the metal substrate made of AlMg6 alloy (roughness $R_{\mathrm{a}}=0.05-0.12 \mu \mathrm{m}$; the roughness was measured by a contactless surface analyzer Micro Measure 3D station), the drop generator (micro-pump, set of syringes and needles), LED spotlight and diffusor, PC and high-speed CMOS video camera (resolution $768 \times 480 \mathrm{px}$, sample rate $8600 \mathrm{fps}$ ). A temperature of the substrate before the drop impingement was $40-500^{\circ} \mathrm{C}$. A height of the drop generator varied by using a linear module. Before the impact onto the wall, a diameter of the drop was $D_{0}=1.75-2.9 \mathrm{~mm}$, a velocity $-U_{0}=0.9-3.1 \mathrm{~m} / \mathrm{s}$. An inaccuracy of determining $D_{0}$ and $U_{0}$ did not exceed $8-10 \%$. 


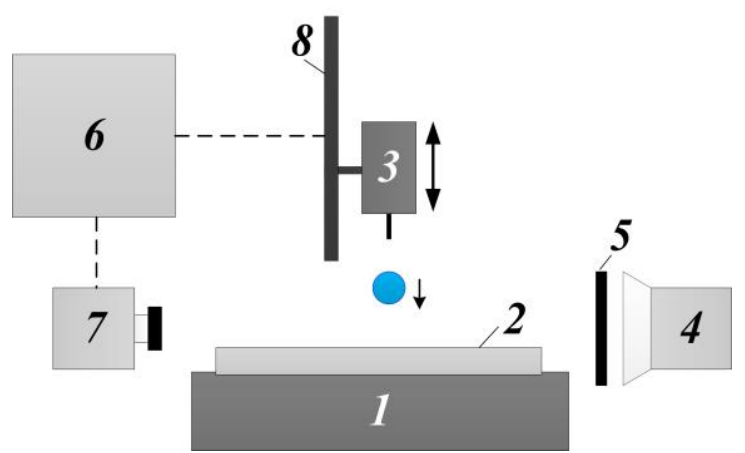

Fig. 1: Scheme of the experimental setup: heating plate (1), substrate (2), drop generator (3), LED spotlight (4), diffusor (5), PC (6), video camera (7), linear module (8).

The experiments were implemented by using winter diesel fuel, WDME fuel and BDME fuel with $0.1 \mathrm{M}$ and $1 \mathrm{M}$ salt concentrations (volume content of a dispersed phase $20 \mathrm{vol} \%$ ).

In order to calculate dimensionless criteria, we measured the properties of the microemulsions (density, viscosity, and surface tension). Table 1 presents the values of the microemulsion properties. The initial temperature of drops was $25^{\circ} \mathrm{C}$. A hydrodynamic regime was defined based on the outcomes of 3-5 tests.

Table 1: Properties of the alternative fuels.

\begin{tabular}{|l|l|l|l|l|}
\hline \multicolumn{1}{|c|}{ Fuel } & $\begin{array}{c}\sigma(\mathrm{N} / \mathrm{m}) \\
\text { at } 25^{\circ} \mathrm{C}\end{array}$ & $\begin{array}{c}\rho\left(\mathrm{kg} / \mathrm{m}^{3}\right) \\
\text { at } 25^{\circ} \mathrm{C}\end{array}$ & $\begin{array}{c}v \cdot 10^{6}\left(\mathrm{~m}^{2} / \mathrm{s}\right) \\
\text { at } 25^{\circ} \mathrm{C}\end{array}$ & $\begin{array}{c}\mu \cdot 10^{3} \\
(\mathrm{~Pa} \cdot \mathrm{s})\end{array}$ \\
\hline WDME & 0.022903 & 893.1 & 45.16 & 40.33 \\
\hline $0.1 \mathrm{M}-\mathrm{BDME}$ & 0.02950 & 895.8 & 46.33 & 41.50 \\
\hline $1 \mathrm{M}-\mathrm{BDME}$ & 0.02883 & 894.6 & 15.35 & 13.73 \\
\hline
\end{tabular}

\section{Results and Discussions}

\subsection{Ways for controlling the WDME fuel stability}

The first way promoting to control temperature ranges of the microemulsion stability is to vary the $S / c S$ ratio in the emulsifier mixture. Through the example of 2-ethylhexanol, we established that an increment of the co-surfactant fraction results in shifting a temperature band of the thermal stability toward lower temperatures without its widening even with an increase of the emulsifier concentration (Fig. 2a). Likely, this result is explained by a reduction of the total HLB (hydrophiliclipophilic balance) of the emulsifier with an increment in the co-surfactant fraction. 


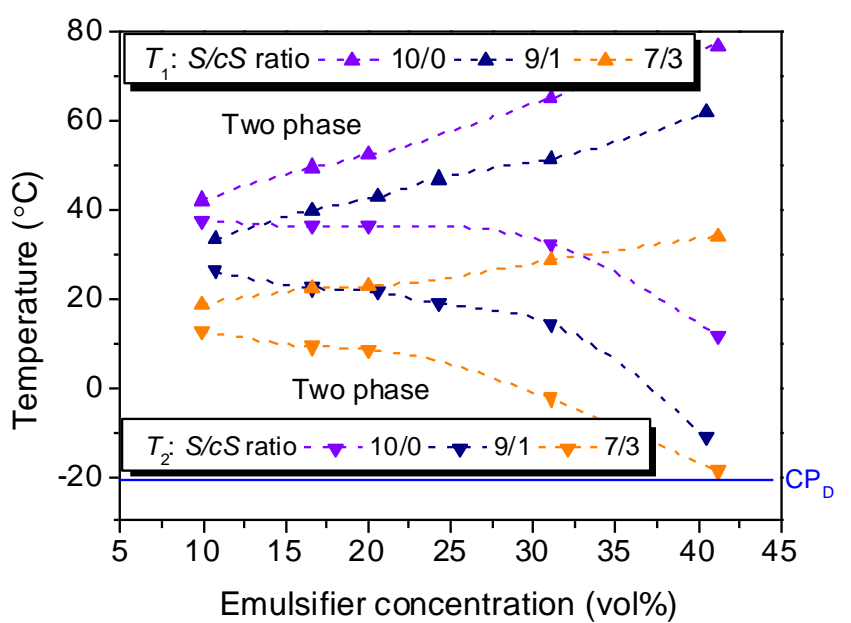

(a)

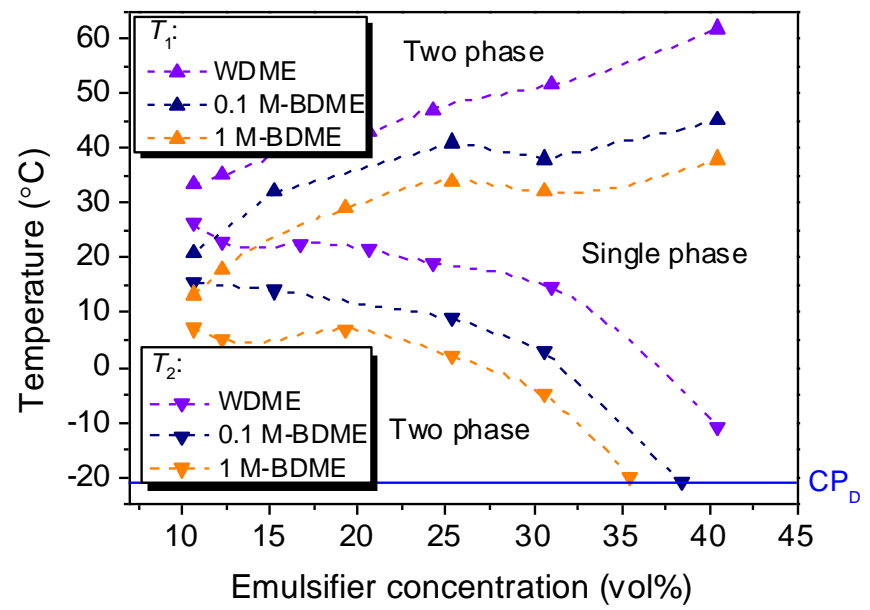

(c)

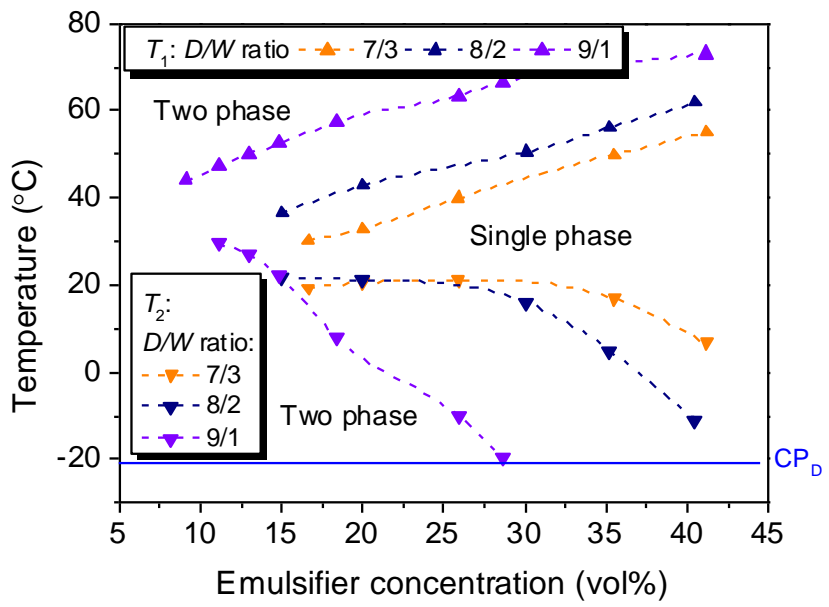

(b)

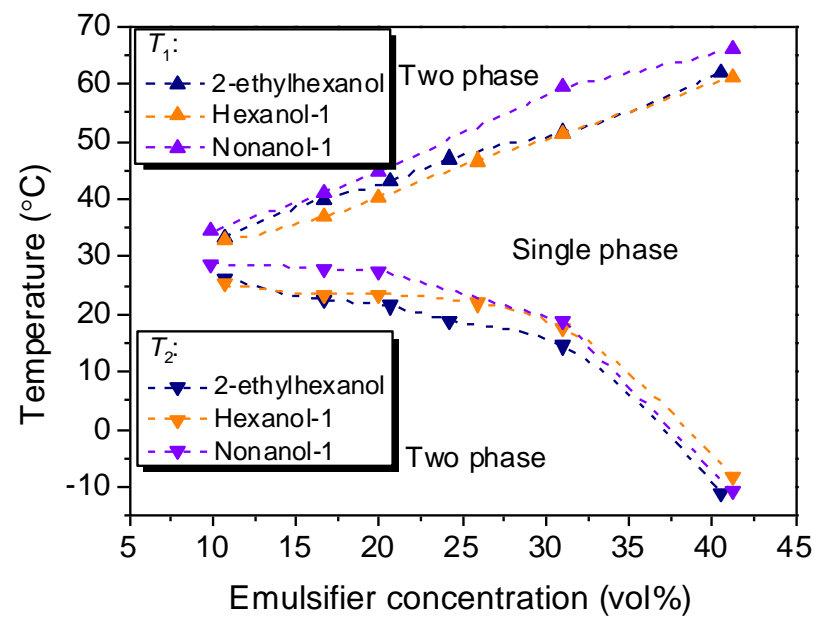

$(d)$

Fig. 2: Ways for controlling the thermal stability of the microemulsion fuel: a variation of the $S / c S(a)$ and $D / W(b)$ ratios, utilization of the ammonium acetate brine as a dispersed phase $(c)$ and various alcohols as a co-surfactant $(d)$; the results depicted in figs. $(a)-(c)$ were obtained using the microemulsions stabilized by 2-ethylhexanol, the results presented in figs. $(a),(c),(d)$ were performed at $D / W=8 / 2 ; \mathrm{CP}_{\mathrm{D}}-$ cloud point of winter diesel fuel.

The second one is to alter the $D / W$ ratio (Fig. $2 b$ ). However, when using this method the temperature ranges of the thermal stability did not shift toward lower temperatures but widened with an increment of the diesel fraction. This trend from a physical standpoint is quite clear. Since the water content decreases, the fuel properties become similar to the neat diesel. Note a sharp drop of the lower CP at a water concentration as little as $10 \mathrm{vol} \%$. The growth of the emulsifier concentration only contributes to a further fall. However, at emulsifier concentrations of $>28$ vol\%, the lower CP attains a value of the $\mathrm{CP}$ for the neat diesel and remains constant.

By analogy with the results in Fig. $2 a$, when using the ammonium acetate brines (0.1 M-BDME and 1 M-BDME) instead of the distilled water, the phenomenon of shifting the temperature ranges of stability without their widening also occurred (Fig. 2c). Note that an increment of the salt concentration enhanced shifting. Such an effect took place due to the surfactant "salting-out" from the water phase. A minimum lower CP of the microemulsion fuel almost attained to the $\mathrm{CP}$ for the winter diesel fuel (see Fig. $2 c$, curves for the lower CPs). Thus, a variation of the $S / c S$ ratio is one more method for controlling the thermal stability of the WDME fuel. 
An important feature is the fact that the nature of co-surfactant influences the thermal stability of the microemulsion fuels insignificantly with an increase of the emulsifier concentration (Fig. $2 d ; S / c S=9 / 1$ ). Nevertheless, the broadest temperature range was observed for the WDME fuels prepared using 2-ethylhexanol. Thus, to stabilize the WDME fuel a a number of alcohols could be applied with a length of the carbon chain from $\mathrm{C}_{5}$ to $\mathrm{C}_{9}$.

\subsection{Drop impact regimes}

The three regimes of the WDME fuel drop impact onto a horizontal heated wall were established (Fig. 3): deposition $(a)$, thermal breakup $(b)$, and rebound $(c)$.

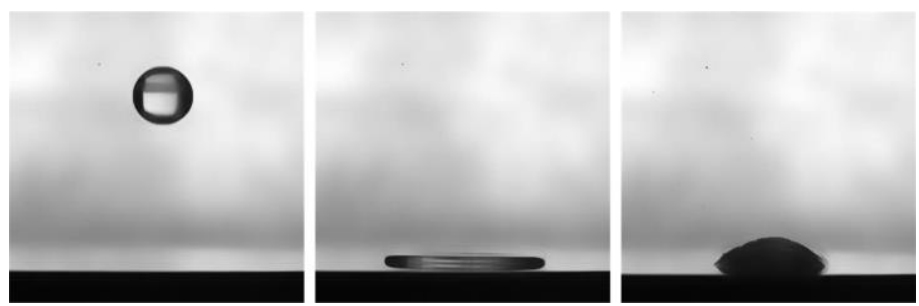

(a)
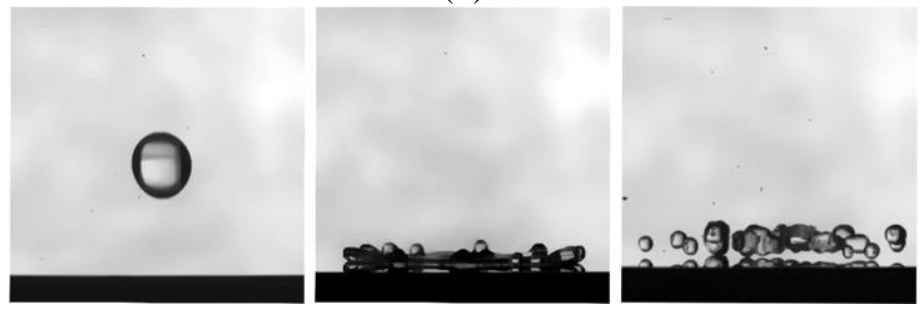

(b)

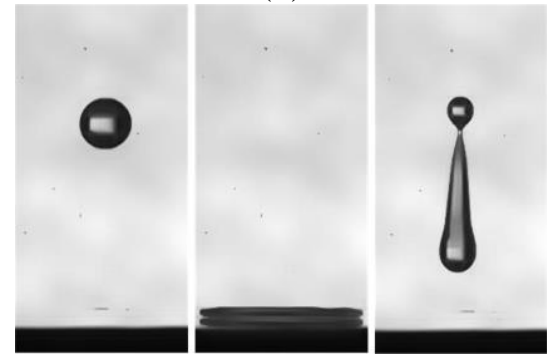

$(c)$

Fig. 3: Hydrodynamic regimes of the WDME fuel drop impact onto a heated wall: deposition $(a)$, thermal breakup $(b)$, rebound $(c)$.

The deposition regime (Fig. 3a) involves vaporization due to convection or bubble boiling depending on wall heating temperature $T_{0}$. The rebound (Fig. $3 c$ ) occurs at higher temperatures $T_{0}$. The thermal breakup regime is characterized by the formation of a great number of secondary drops. This process results from rim disintegration of the spreading drop. Size of the secondary drops was almost uniform. However, it depends on the drop velocity before the impact $U_{0}$. The higher velocity $U_{0}$, the smaller dimensions of the emerging drops will be.

To reveal the impact parameters characterizing the thermal breakup / rebound transition, we implemented the experiments at $T_{0}=320{ }^{\circ} \mathrm{C}$ and various $D_{0}$ and $U_{0}$. The main conclusion made according to the dependence depicted in Fig. 4 lies in the fact that the transition to the thermal breakup regime cannot be characterized only by the Weber number, i.e. inertia forces [9]. More likely, the transition between the hydrodynamic regimes mainly depends on the viscosity force. A high viscosity of the WDME fuel results from an occurrence of the dispersed phase. Fig. 4 shows that for the liquids with far different viscosities it is complex to find out either critical (threshold) Reynolds numbers or critical Weber ones. 


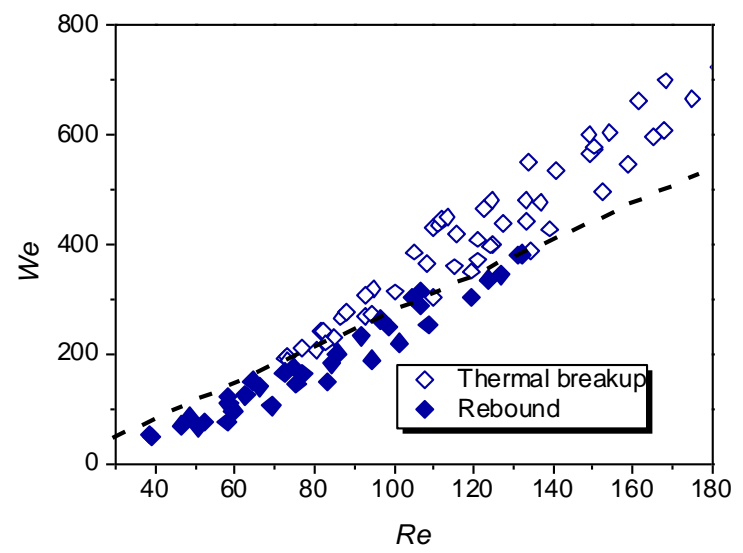

Fig. 4: Map of thermal breakup / rebound transition for the WDME fuel drops in the form of the $W e(R e)$ dependence $\left(T_{0}=320^{\circ} \mathrm{C}\right)$.

The experimental results show that the fabricated WDME fuels tend to a hydrodynamic fragmentation when impinging onto a wall. The findings can be used in practice, e.g., when a formation of the air-fuel mixture occurs in combustion chambers of various IC-engines and power generation systems.

\section{Conclusion}

The study presents several ways for controlling temperature ranges of the thermal stability, namely a variation of the surfactant / co-surfactant and diesel / water ratios, utilization of the ammonium acetate brines (with a required concentration) as a dispersed phase and various alcohols as a co-surfactant. These methods allow the fabrication of the microemulsion fuel stable at temperatures from $-21^{\circ} \mathrm{C}$ to $73^{\circ} \mathrm{C}$. Varying of $S / c S$ and $D / W$ ratios and using of the ammonium acetate brines as a dispersed phase are convenient tools for controlling the microemulsion fuel stability in a wide temperature range, namely for shifting and widening the cloud points. In addition, the research explores the hydrodynamic regimes of the WDME fuel drop impact onto a horizontal heated wall. The corresponding maps of the impact regimes are plotted to show relationships between inertia, viscosity and surface tension forces.

\section{Acknowledgements}

The study was funded by the Russian Science Foundation (18-73-00083).

\section{References}

[1] X. T. Tran and J. I. Ghojel, "Impact of introducing water into the combustion chamber of diesel engines on emissions An overview," in Proceedings of 5th Asia-Pacific Confeferce on Combustion, Adelaide, Georgia, 2005, pp. 233-236.

[2] A. Lif and K. Holmberg, "Water-in-diesel emulsions and related systems," Adv. Colloid Interface Sci., vol. 123-126, pp. 231-239, 2006.

[3] O. Armas, R. Ballesteros, F. J. Martos, J. R. Agudelo, "Characterization of light duty Diesel engine pollutant emissions using water-emulsified fuel,” Fuel., vol. 84, no. 7-8, pp. 1011-1018, 2005.

[4] I. Kayali, M. Karaein, K. Qamhieh, S. Wadaah, W. Ahmad, U. Olsson, "Phase Behavior of Bicontinuous and Water/Diesel Fuel Microemulsions Using Nonionic Surfactants Combined with Hydrophilic Alcohol Ethoxylates," J. Dispers. Sci. Technol., vol. 36, no. 1, pp. 10-17, 2015.

[5] I. Kayali, M. Karaeen, W. Ahmed, K. Qamhieh, U. Olsson, "Alternative Diesel Fuel: Microemulsion Phase Behavior and Combustion Properties," J. Dispers. Sci. Technol., vol. 37, no. 6, pp. 894-899, 2016.

[6] B. Acharya and S. Dash, "Tuning commercial diesel to microemulsified and blended form: phase behavior and implications," J. Dispers. Sci. Technol., vol. 0, no. 0, pp. 1-10, 2018. 
[7] R. Najjar and S. Heidari, "Modified diesel prepared by stabilization of water as nanodroplets in diesel/colza oil blend: Study of phase behavior and affecting parameters," Fuel., vol. 214, pp. 497-504, 2018.

[8] J. Breitenbach, J. Kissing, I. V. Roisman, C. Tropea, "Characterization of secondary droplets during thermal atomization regime," Exp. Therm. Fluid Sci. vol. 98, pp. 516-522, 2018.

[9] I. V. Roisman, J. Breitenbach, C. Tropea, "Thermal atomisation of a liquid drop after impact onto a hot substrate," J. Fluid Mech., vol. 842, pp. 87-101, 2018. 